\title{
Low monocyte HLA-DR expression as an indicator of immunodepression in very low birth weight infants
}

\author{
Anniina Palojärvi', Jari Petäjä', Sanna Siitonen², Cecilia Janér ${ }^{1}$ and Sture Andersson ${ }^{1}$
}

BACKGROUND: As a protective response to an inflammatory stimulus, the antigen-presenting molecules (human leukocyte antigen-DR (HLA-DR)) on monocytes are downregulated. If severe, the response may lead to immunodepression or immunoparalysis, associated with an increased rate of morbidity and mortality in adults. In very low birth weight (VLBW) infants, birth and intensive care present major immunological challenges.

METHODS: We measured monocyte HLA-DR expression by flow cytometry and determined 13 plasma cytokines in 56 VLBW infants (gestational age (GA): 23.7-31.8wk) and 25 controls (GA: 34.1-41.4wk).

RESULTS: HLA-DR expression decreased postnatally both in VLBW and in control infants. In VLBW infants, GA and respiratory distress syndrome (RDS) both showed associations with HLA-DR nadir on day 3, when $45 \%$ of them met the criteria of immunodepression. HLA-DR expression was lower in those infants subsequently developing infection (74 vs. $49 \%$ (day 3) and 85 vs. $68 \%$ (day 7); both $P=0.002$ ). Interleukin (IL)- 6 on day 1 was a predictor of the HLA-DR nadir.

CONCLUSION: VLBW infants are in a state of immunodepression postnatally. This immunodepression correlated with GA and was a predisposing factor for late infections. The downregulation of HLA-DR during RDS probably indicates an RDSinduced antigen load on the immune system.

N ewborn infants, particularly those born preterm, face immunological challenges after birth because of the developmental stage of their immune systems (1-3). Important elements in innate immunity responses are circulating monocytes. Monocytes express human leukocyte antigen-DR (HLA-DR) molecules, which are responsible for antigen presentation to $\mathrm{T}$ cells, thereby presenting antigens to adaptive immune system cells. Cytokines released from monocytes mediate responses to other cells and activate complement and coagulation cascades (4).

In acute illness or trauma, an inflammatory burst may lead to the downregulation of the immune system in order to protect the organism from overwhelming systemic inflammation. This downregulation, the compensatory anti-inflammatory response syndrome (CARS), either acts as a protective mechanism or, if prolonged or extensive, becomes harmful in adults. Decrease in HLA-DR expression is indicative for CARS (4).
Persistent low $(<60 \%)$ HLA-DR expression on monocytes is termed immunodepression or, if monocyte HLA-DR expression is $<30 \%$, immunoparalysis $(4,5)$. In adults, immunoparalysis and immunodepression are associated with the risk of infections and death (6-8). In critically ill surgical patients, low HLA-DR is considered causative in the development of surgical infections (9).

In patients with trauma, rapid return of HLA-DR to $>75 \%$ correlates with good recovery. By contrast, infected trauma patients show a delayed return to HLA-DR $>75 \%$ and a persistent low HLA-DR of $20-30 \%$ even correlates with mortality (10). In adult patients with septic shock, survivors show a recovery of HLA-DR expression on days 3-4. An HLA-DR expression $<30 \%$ on days $3-4$ is independently associated with mortality (11). In pediatric patients with cardiopulmonary bypass, HLA-DR expression $<60 \%$ is associated with an increased risk of a systemic inflammatory response or of sepsis later in intensive care (12).

The available data on HLA-DR expression in infants are sparse and fragmentary. In cord blood, monocyte HLA-DR expression is lower than that in healthy adults. Moreover, in preterm infants, cord blood monocytes show significantly lower HLA-DR expression than those of control infants (2). Respiratory distress syndrome (RDS) is a strongly inflammatory state $(13,14)$, and in infants with RDS or infection, HLA-DR expression is low $(15,16)$.

Immunodepression or immunoparalysis has not been described in preterm infants during their early postnatal course. We studied CARS, measured as monocyte HLA-DR expression, during the first postnatal week in very low birth weight (VLBW) infants and evaluated its relationship with immaturity, inflammation, morbidity, and long-term outcome. We hypothesized that in VLBW infants, immunodepression is a factor in the development of postnatal morbidities.

\section{RESULTS}

\section{HLA-DR Expression}

The proportion of HLA-DR-positive monocytes vs. all monocytes (HLA-DR expression) over the observation period in VLBW and control infants is shown in Figure 1. In the VLBW infants, median monocyte HLA-DR expression in cord blood 


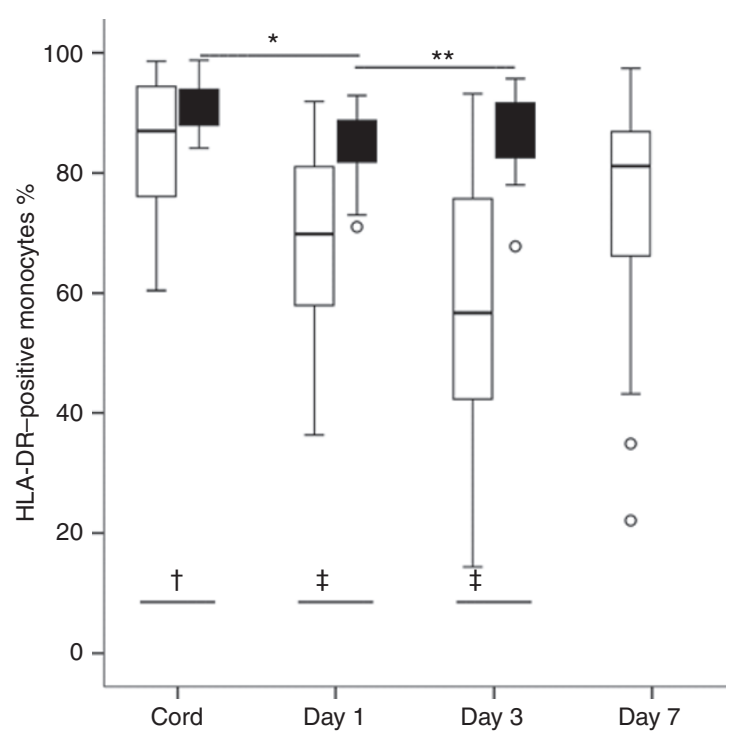

Figure 1. Monocyte HLA-DR\% in VLBW (white bars) and control infants (black bars). Control infants showed a temporary decline in HLA-DR expression, with return to cord values on day 3 (Friedman $P=0.018$ ), whereas in VLBW infants, the decline was steep, with its nadir on day 3 (Friedman $P<0.001$ ). ${ }^{*} P<0.001,{ }^{*} P=0.028,{ }^{\dagger} P=0.04,{ }^{*} P<0.001$. Lines represent medians; boxes, 50th percentiles; and whiskers, range (\%). Open circles represent individual values more than 1.5 times the interquartile range outside the respective median. Samples available for each time point: cord, 26 (VLBW) and 20 (control); day 1, 51 (VLBW) and 18 (control); day 3, 46 (VLBW) and 12 (control); day 7, 40 (VLBW) and 0 (control). HLA-DR, human leukocyte antigen-DR; VLBW, very low birth weight.

Table 1. Correlations of HLA-DR and clinical parameters

\begin{tabular}{|c|c|c|c|c|}
\hline & Gestational age & $\mathrm{PaO}_{2} / \mathrm{FiO}_{2}$ & SNAP-II & SNAPPE-II \\
\hline HLA-DR, cord & $\begin{array}{l}r^{2}=0.167 \\
P=0.038 \\
n=26\end{array}$ & $\begin{array}{l}r^{2}=-0.266 \\
P=0.007 \\
n=26\end{array}$ & NS & NS \\
\hline HLA-DR, day 1 & $\begin{array}{l}r^{2}=0.106 \\
P=0.020 \\
n=51\end{array}$ & NS & NS & NS \\
\hline HLA-DR, day 3 & $\begin{array}{l}r^{2}=0.530 \\
P<0.001 \\
n=46\end{array}$ & $\begin{array}{l}r^{2}=0.087 \\
P=0.047 \\
n=51\end{array}$ & NS & NS \\
\hline HLA-DR, day 7 & $\begin{array}{l}r^{2}=0.482 \\
P<0.001 \\
n=40\end{array}$ & NS & $\begin{aligned} r^{2} & =-0.142 \\
P & =0.016 \\
n & =40\end{aligned}$ & $\begin{aligned} r^{2} & =-0.148, \\
P & =0.013, \\
n & =40\end{aligned}$ \\
\hline
\end{tabular}

$\mathrm{FiO}_{2}$, fraction of inspired oxygen; HLA-DR, human leukocyte antigen-DR; NS, not significant; SNAP-II, Score for Neonatal Acute Physiology-II; SNAPPE-II, SNAP Perinatal Extension-II.

was $87 \%$, which decreased after birth to a nadir of $57 \%$ on day 3 (Figure 1). In comparison with control infants, monocyte HLA-DR expression in VLBW infants was significantly lower at all time points (Figure 1). Furthermore, significant correlations existed between gestational age (GA) and HLA-DR at all time points (Figure 2; Supplementary Figure S1 online; Table 1). Of the VLBW infants, 14 on day 1, 22 on day 3 , and 5 on day 7 met the criteria for immunodepression (HLA-DR expression on $30-60 \%$ of monocytes). Three infants on day 3 and one on day 7 met the criteria of immunoparalysis (HLA-DR expression on $<30 \%$ of monocytes). Monocyte

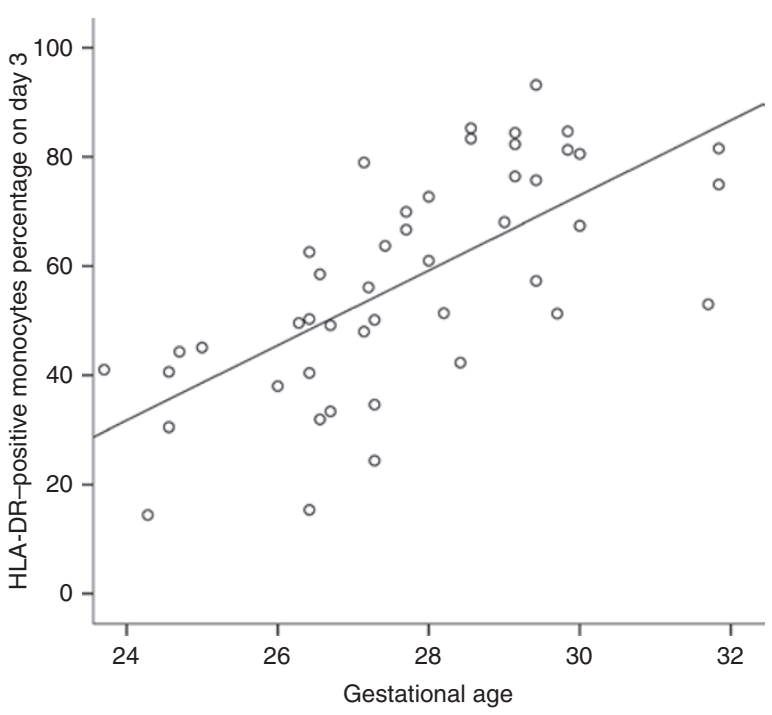

Figure 2. Correlation between gestational age (GA) (wk) and HLA-DR on day $3, n=46 ; r^{2}=0.530 ; P<0.001$. For the control infants (GA: 34-41 wk), 12 samples were available from day 3 , and the correlation between GA and HLA-DR was not significant $\left(r^{2}=0.02 ; P=0.678\right)$. However, on day 1 , on the nadir of HLA-DR in control infants, a positive correlation appeared between GA and HLA-DR $\left(r^{2}=0.25 ; P=0.045 ; n=17\right)$. HLA-DR, human leukocyte antigen-DR.

CD11b expression was determined in parallel for control purposes. This monocyte surface component behaved qualitatively differently, showing in cord monocytes a median of 3,780 molecules per cell: 5,525 molecules per cell on day 1; 4,361 molecules per cell on day 3; and 3,080 molecules per cell on day 7 .

\section{Clinical Morbidity vs. HLA-DR}

To evaluate the potential clinical implications of the immunodepression or immunoparalysis in VLBW infants, we studied the association of HLA-DR expression with the presence or subsequent development of RDS, intraventricular hemorrhage (IVH), bronchopulmonary dysplasia (BPD), and infection (Table 2). Association of HLA-DR\% with all these usual morbidities of the VLBW infants was evident because significant correlations appeared for each postnatal complication. IVH showed the lowest HLA-DR as generally coinciding with the appearance of the lesion, and low HLA-DR expression seemed to precede the appearance of late infections.

Infants with subsequent infections had lower GA than did infants without late infections ( 27 vs. $29 \mathrm{wk} ; P=0.001$ ). Of special interest, in VLBW infants, subsequent development of BPD was associated with low HLA-DR on day 3 (Table 2), i.e., at the nadir of HLA-DR expression (Figure 1). We also evaluated early respiratory morbidity vs. HLA-DR using the lowest $\mathrm{PaO}_{2}$ /fraction of inspired oxygen $\left(\mathrm{FiO}_{2}\right)$ during the first $12 \mathrm{~h}$ of life (Table 1). As composite measures of morbidities, we used the Score for Neonatal Acute Physiology-II (SNAP-II) and SNAP Perinatal Extension-II (Table 1). High scores on the SNAP-II and SNAP Perinatal Extension-II were associated with prolongation of low HLA-DR expression (negative correlation with HLA-DR on day 7; Table 1). 
Table 2. HLA-DR in infants with and without RDS, BPD, IVH, and in infants developing infection after day 3

\begin{tabular}{|c|c|c|c|c|c|c|c|c|c|c|c|c|c|}
\hline & \multicolumn{3}{|c|}{ RDS } & \multicolumn{3}{|c|}{ BPD } & \multicolumn{3}{|c|}{ IVH } & \multicolumn{4}{|c|}{ Infection } \\
\hline & Yes & No & & Yes & No & & Yes & No & & Yes, BC+ & Yes, BC- & No & \\
\hline & $\begin{array}{c}n=35 \\
(\%)\end{array}$ & $\begin{array}{c}n=21 \\
(\%)\end{array}$ & $P$ value & $\begin{array}{c}n=16 \\
(\%)\end{array}$ & $\begin{array}{c}n=40 \\
(\%)\end{array}$ & $P$ value & $\begin{array}{c}n=19 \\
(\%)\end{array}$ & $\begin{array}{c}n=37 \\
(\%)\end{array}$ & $P$ value & $\begin{array}{c}n=17 \\
(\%)\end{array}$ & $\begin{array}{c}n=14 \\
(\%)\end{array}$ & $\begin{array}{c}n=25 \\
(\%)\end{array}$ & $P$ value \\
\hline HLA-DR, cord & 91 & 76 & 0.01 & 91 & 85 & NS & 85 & 88 & NS & 86 & 82 & 90 & NS \\
\hline HLA-DR, day 1 & 68 & 70 & NS & 68 & 70 & NS & 68 & 70 & NS & 70 & 62 & 71 & NS \\
\hline
\end{tabular}

HLA-DR values are median percentages of HLA-DR-positive monocytes.

BC, blood culture; BPD, bronchopulmonary dysplasia; HLA-DR, human leukocyte antigen-DR; IVH, intraventricular hemorrhage; NS, not significant; RDS, respiratory distress syndrome.

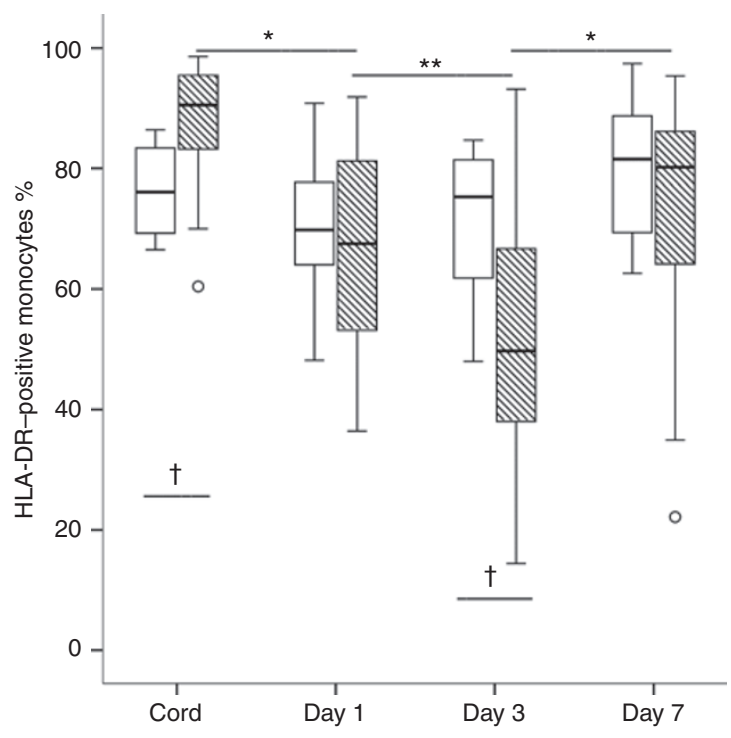

Figure 3. Monocyte HLA-DR expression in RDS (striped bars) and NoRDS infants (white bars). RDS infants had higher cord HLA-DR expression than did NoRDS infants and postnatally a steep decline. The HLA-DR nadir in RDS infants was on day 3 (Friedman $P<0.001$ ), whereas in NoRDS infants, the recovery of HLA-DR had already occurred on day 3 (Friedman $P=0.003) .{ }^{*} P>0.001,{ }^{* *} P=0.001,{ }^{+} P=0.01$. Lines represent medians; boxes, 50th percentiles; and whiskers, range (\%). Open circles represent individual values more than 1.5 times the interquartile range outside the respective median. Samples available for each time point: cord, 7 (NoRDS) and 19 (RDS); day 1, 17 (NoRDS) and 34 (RDS); day 3, 16 (NoRDS) and 30 (RDS); day 7, 12 (NoRDS) and 28 (RDS). HLA-DR, human leukocyte antigen-DR; NoRDS, without respiratory distress syndrome; RDS, respiratory distress syndrome.

The infants with RDS had lower GA (27.4 vs. 28.9 wk; $P=$ 0.012 ) and lower birth weight ( 985 vs. $1,121 \mathrm{~g} ; P=0.035$ ) than did infants without RDS (NoRDS). HLA-DR expression of infants with RDS as compared with that of NoRDS infants was higher in cord blood but significantly lower at the day 3 nadir (Figure 3 ).

We studied the performance of low HLA-DR expression on day 3 as a predictor of development of infection, BPD, or IVH that coincided or appeared after day 3 . The subgroups were appearance of late infection $(n=29), \operatorname{BPD}(n=14)$, and IVH appearing or worsening at 3- or 7-d scans $(n=13)$. As seen in Figure 4, all the 29 infections were diagnosed at least $4 \mathrm{~d}$ after birth, and 26 of them appeared at least $7 \mathrm{~d}$ postpartum. Therefore, for infections and BPD, the receiver operating

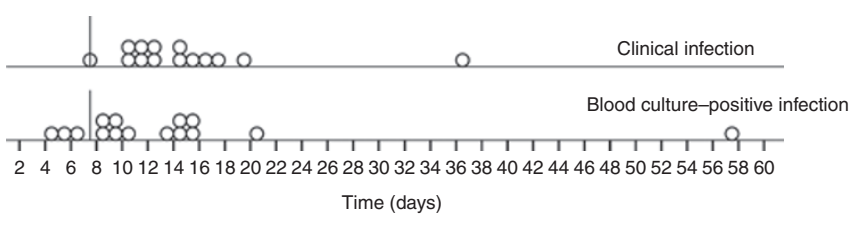

Figure 4. Chronological distribution of infections in VLBW infants. Infections after day 3 are shown (two infants with positive blood culture on day 1 were excluded). Three blood culture-positive infections appeared before day 7; one clinical infection appeared on day 7. The attending clinicians, blind to the study, made the diagnosis of infection, on the basis of symptoms and signs, C-reactive protein, and leukocytes. Blood cultures were obtained from all infection patients. VLBW, very low birth weight.

characteristic analysis of day-3 HLA-DR expression (the HLA-DR nadir) vs. clinical complications addressed true predictive properties of HLA-DR determination, whereas for IVH, part of the bleeding coincided with the HLA-DR measurement. We chose to use the threshold of HLA-DR $<60 \%$ on day 3 , which then gave an area under the curve of 0.761 (confidence interval: 0.614-0.909) for infections, 0.778 (confidence interval: 0.634-0.923) for BPD, and 0.701 (confidence interval: 0.525-0.877) for IVH (Figure 5).

\section{Cytokines vs. HLA-DR}

We measured the panel of 13 cytokines (the concentrations of cytokines are shown in Supplementary Table S1 online) and analyzed correlations of each cytokine against the coinciding or subsequent levels of HLA-DR expression in our VLBW infants. In this analysis, interleukin (IL)-6 and IL-8 each showed a significant correlation with HLA-DR.

In VLBW infants, IL-6 and IL-8 concentrations peaked on day 1 and then returned to near-cord levels (Figure 6). Negative correlations appeared between IL-8 and HLA-DR in cord blood and on days 3 and 7 (Figure 6). Similarly, negative correlations appeared between IL- 6 and HLA-DR in cord blood and on day 3 (Figure 6). Furthermore, IL-6 on day 1 correlated with HLA-DR on day $3\left(r^{2}=-0.251 ; P=0.005\right)$. This association was further confirmed in the regression analysis in which IL-6 on day 1 proved to be an independent predictor for the HLA-DR nadir. Of note, no association were found between HLA-DR and concentrations of interferon- $\gamma$ or tumor necrosis factor- $\alpha$. Likewise, HLA-DR did not correlate with the anti-inflammatory 


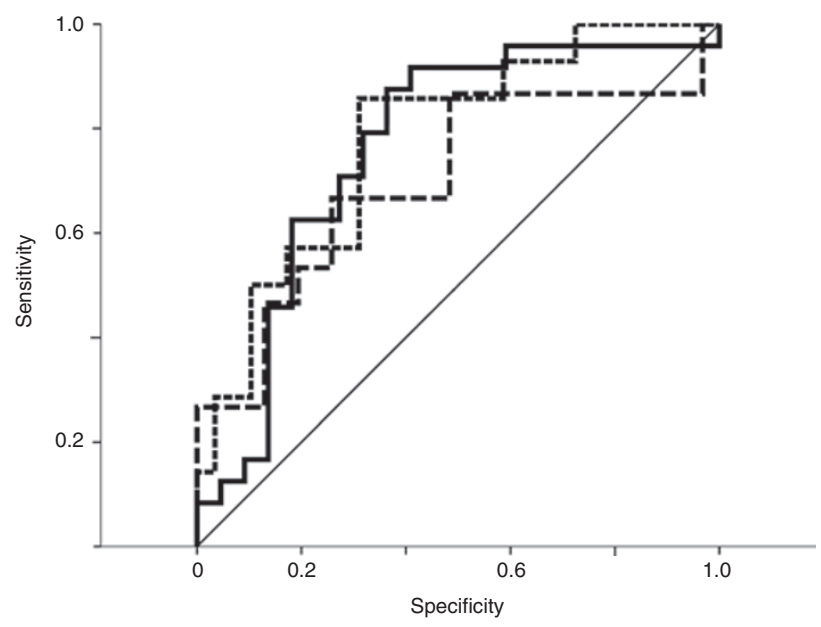

Figure 5. HLA-DR on day 3 as a predictor of infection (solid line), BPD (dotted line), or IVH (dashed line). ROC analysis was performed by free distribution of HLA-DR values. Using a cutoff of $60 \%$ of HLA-DR expression, previously used as the criteria for immunodepression in pediatric and adult patients alike, the sensitivity of HLA-DR to detect/predict infection was $75 \%$, to detect/predict BPD was $86 \%$, and to detect/predict IVH was $69 \%$. The corresponding specificities of the test were 68,65 , and $51 \%$. BPD bronchopulmonary dysplasia; HLA-DR, human leukocyte antigen-DR; IVH, intraventricular hemorrhage; ROC, receiver operating characteristic.

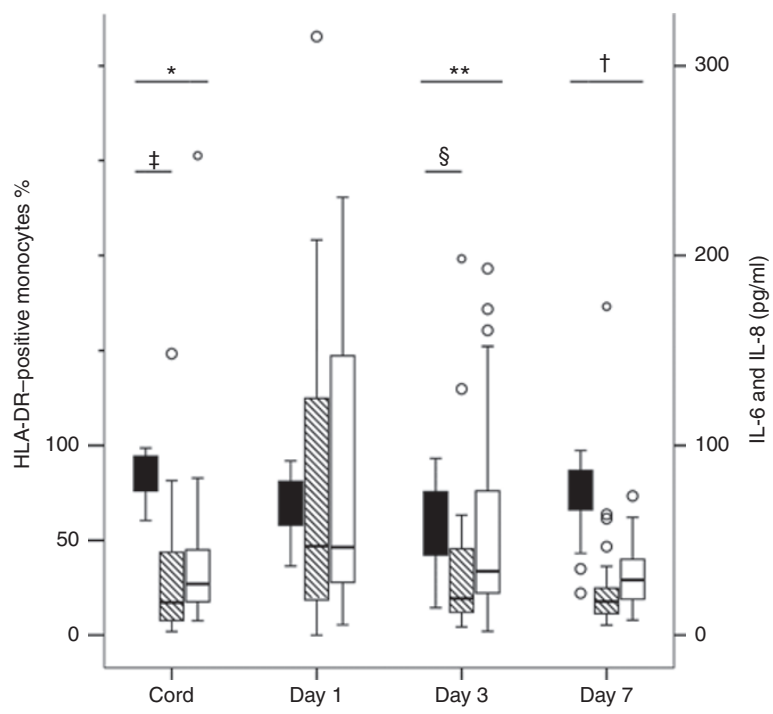

Figure 6. HLA-DR\% (black bars, on the left side of $y$-axis), IL-6 (striped bars, on the right side of $y$-axis), and IL-8 (pg/ml) (white bars, on the right side of $y$-axis) in plasma of VLBW infants. Significant correlations with the corresponding HLA-DR level, ${ }^{*} r^{2}=-0.387, P=0.008, n=14 ; *{ }^{*} r^{2}=-0.393, P$ $=0.001, n=26 ;{ }^{\dagger} r^{2}=-0.245, P=0.005, n=30 ;{ }^{*} r^{2}=-0.334, P=0.03, n=14$; ${ }^{8} r^{2}=-0.165, P=0.048, n=24$. On day 7, IL-6 nearly reached significance: $r^{2}$ $=-0.119, P=0.053, n=32$. Lines represent medians; boxes, 50th percentiles; and whiskers, range (\% or $\mathrm{pg} / \mathrm{ml})$. Open circles represent individual values more than 1.5 times the interquartile range outside the respective median. Two outliers ( $>350 \mathrm{pg} / \mathrm{ml}$ ) of cord IL-6 values and eight outliers of day 1 IL-6 values (350-2,000 pg/ml) are not shown. HLA-DR, human leukocyte antigen-DR; IL, interleukin; VLBW, very low birth weight.

cytokines, including IL-4 and IL-10. Analyses of HLA-DR vs. the remaining measured cytokines (namely, IL-1 $\beta$, IL-2, IL-5, IL-7, IL-12, IL-13, and granulocyte macrophage -colonystimulating factor $-\beta$ ) were unrewarding.
Table 3. Clinical characteristics of VLBW infants

\begin{tabular}{|c|c|}
\hline & Patients, $n(\%)$, total 56 \\
\hline \multicolumn{2}{|l|}{ Prenatal factors } \\
\hline \multicolumn{2}{|l|}{ Antenatal betamethasone } \\
\hline Two doses & $54(96)$ \\
\hline One dose & $2(4)$ \\
\hline Preeclampsia & $16(28)$ \\
\hline Clinical chorioamnionitis & $15(27)$ \\
\hline Preterm rupture of membranes $>24 \mathrm{~h}$ & $11(19)$ \\
\hline \multicolumn{2}{|l|}{ Delivery } \\
\hline Vaginal & $16(28)$ \\
\hline Cesarean section & $40(71)$ \\
\hline \multicolumn{2}{|l|}{ Postnatal factors } \\
\hline \multicolumn{2}{|l|}{ Gender } \\
\hline Female & $19(34)$ \\
\hline Male & $37(66)$ \\
\hline Gestational age (weeks; range, mean, SD) & $23.7-31.9 ; 28(1.9)$ \\
\hline Birth weight (g; range, mean, SD) & $605-1,500 ; 1,034(220)$ \\
\hline Twins & $16(28)$ \\
\hline \multicolumn{2}{|l|}{ Apgar score } \\
\hline $1 \mathrm{~min}$ & $5(1-9)$ \\
\hline $5 \mathrm{~min}$ & $7(1-9)$ \\
\hline Umbilical artery pH (mean, SD) & $7.30(0.07)$ \\
\hline \multicolumn{2}{|l|}{ Surfactant doses } \\
\hline None & $12(21)$ \\
\hline One dose & $18(32)$ \\
\hline Two doses & $12(21)$ \\
\hline Three or more & $14(25)$ \\
\hline Prophylactic surfactant & $41(72)$ \\
\hline Intubation immediately after birth & $41(72)$ \\
\hline Intubated infants, total & $44(79)$ \\
\hline Nasal CPAP only & $12(21)$ \\
\hline Duration of ventilatory care (range) & $1 \mathrm{~h}-91 \mathrm{~d}$ \\
\hline RDS & $35(63)$ \\
\hline Only in CPAP & $3 / 35$ \\
\hline BPD & $16(28)$ \\
\hline \multicolumn{2}{|l|}{ Neonatal infection } \\
\hline Clinical infection, blood culture negative & $14(25)$ \\
\hline Blood culture positive & $17(30)$ \\
\hline Late infection, onset after age $72 \mathrm{~h}$ & $29 / 31$ \\
\hline Late infection, onset after age $7 \mathrm{~d}$ & $25 / 31$ \\
\hline IVH & $19(34)$ \\
\hline
\end{tabular}

BPD, bronchopulmonary dysplasia; CPAP, continuous positive airway pressure; IVH intraventricular hemorrhage; RDS, respiratory distress syndrome; VLBW, very low birth weight.

\section{DISCUSSION}

The current results demonstrate that VLBW infants undergoing intensive care show a progressively developing monocyte HLA-DR expression pattern qualitatively similar to that in CARS seen in adults after strong initial activation of the 
immune system. However, caution is merited before taking these observations to indicate CARS in the newborns, given that the most complicated physiology of the postnatal adaption per se might well involve rapid changes in the innate immune system. The facts that the VLBW infants almost invariably and immediately postnatally develop, especially respiratory, morbidities (17) associated with inflammatory activation, i.e., requirement of the initial immune activation before CARS, and that the control infants without signs of respiratory morbidity showed a less pronounced pattern during the first week of life of decreasing HLA-DR, lead us to believe that a transient CARS is, indeed, an integral part of the early postnatal course of a sick VLBW infant. Our results cannot, however, be extrapolated to healthy term infants, and the true interplay between physiological birth, early postnatal adaption, and the innate immune system is beyond the scope of the current study.

The magnitude of immunodepression is strongly associated with the level of immaturity. It remains impossible to conclude whether this phenomenon presents GA-dependent differences in the developmental stage of the innate immune system, the well-known GA dependency of major neonatal morbidities, or both. However, this observation raises the intriguing possibility that if CARS is a causative factor for several adverse outcomes, as suggested for several groups of adult patients, the developmental properties of the neonatal innate immune system may prove to be a more comprehensive determinant of the general course of VLBW infants than is generally recognized.

In the absence of relevant neonatal reference values, we adopted from the adult literature the qualitative terms immunodepression and immunoparalysis, with the corresponding cutoffs of 60 and $30 \%$ for HLA-DR expression $(4,5)$ to facilitate categorizing our data. We find it demonstrative that at the HLA-DR nadir on day 3, up to $45 \%$ of the VLBW infants met these criteria for immunodepression or immunoparalysis, and not all infants indicated recovery with HLA-DR above these cutoffs by day 7. By contrast, the morbidity and mortality risk scores SNAP-II and SNAP Perinatal Extension-II both showed an association with low HLA-DR expression on day 7, suggesting that prolonged immunodepression or immunoparalysis may play a role in preterm infants' morbidity and mortality. Our results are novel in demonstrating a clear association between decreasing HLA-DR expression and overall level of morbidity of the preterm infant and are also consistent with the literature.

Our VLBW infants subsequently developing infection during intensive care showed lower proportions of HLA-DR-positive monocytes on days 3 and 7. With a cutoff of HLA-DR expression $<60 \%$ on day 3 (12), the sensitivity of low HLA-DR to predict infection was $75 \%$ and its specificity was $68 \%$ (Figure 5). By contrast to the findings of Allen et al. in patients with pediatric cardiopulmonary bypass, infection did not become an independent predictor for CARS. Given that the concept of CARS involves the initial activation of the immune system, this difference could logically be explained by assuming that in VLBW infants, the sequel might rather be early morbidity, including RDS $(14,17)$, causing CARS, which then could be one causative factor in developing infections. The low GA, being independently associated with the low HLA-DR expression, would logically fortify this adverse feedback loop.

Evaluation of the effect of CARS on mortality in the current study was not feasible because of the low mortality rate. However, low HLA-DR was associated with higher incidence of BPD and IVH. In adjusting for GA, IVH lost its role as an independent factor, whereas independent associations still existed between the lowest HLA-DR expression and BPD and infections. It is well known that the more premature a VLBW infant is, the more prone that infant is to infections. We suggest that GA-dependent immunodepression may be one central mediator of the link between immaturity and risk of infection.

RDS and early respiratory mortality measured by initial $\mathrm{PaO}_{2} / \mathrm{FiO}_{2}$ were accompanied by a steep decline in and a persistent low HLA-DR, indicating that pulmonary morbidity may be a pathogenic factor associated with CARS. This is in line with findings that demonstrate that mechanical ventilation induces systemic inflammation in preterm infants (17). Linear regression analysis showed that RDS and GA were both independent predictors for low HLA-DR on day 3. Therefore, RDS seems to be a patient-specific clinical factor adding to the burden of prematurity itself in pushing the individual infant toward depressed innate immunity.

Studies show that several cytokines may cause or contribute to low HLA-DR $(8,18,19)$. In accordance with the interpretation of reduced HLA-DR expression as a mechanism adaptive to an overwhelming burst of inflammatory stimuli, we found significant negative associations among IL-6, IL-8, and HLA-DR. Linear regression analysis showed that, of all the cytokines, IL-6 on day 1 was an independent predictor of the HLA-DR nadir. We found, however, no correlation between HLA-DR and the anti-inflammatory cytokines, IL-4, IL-10, and IL-13. Overall, the data suggest that, as compared with those in adults, in the VLBW infant proinflammatory cytokines play a more central role in the pathogenesis of CARS. The fact that VLBW infants showed low HLA-DR but high proinflammatory cytokines may be an important element in maintaining innate immunity responses despite low HLA-DR. In adult studies, survivors of septic shock showed an upregulation of proinflammatory gene transcriptions but nonsurvivors did not (20).

The molecular mechanisms behind CARS cannot be elucidated in this study. CARS was evident within $24 \mathrm{~h}$ after birth, with its nadir reached in $72 \mathrm{~h}$. This behavior and associations of CARS postnatally are in accordance with adult and pediatric studies (6-12). Therefore, in adults and VLBW infants, the molecular mechanisms might also have similarities. Internalization of HLA-DR molecules could be one mechanism for the rapid HLA-DR decline (21). In adults, IL-10 reduced HLA-DR expression by inhibiting exocytosis and the recycling of existing HLA-DR (22). One relevant mechanism in VLBW infants could be that high HLA-DR-expressing monocytes are preferentially recruited on the site of new antigens, e.g., lungs, and a new pool of monocytes released from bone marrow might express HLA-DR to a lesser extent. 


\section{Conclusions}

VLBW infants have low HLA-DR expression on monocytes during their first postnatal week comparable with the criteria of immunodepression or immunoparalysis in adult patients. Low HLA-DR expression is associated with immaturity and pulmonary morbidity and was lower in infants developing complications, especially infections.

\section{METHODS}

\section{Patients and Controls}

We enrolled 56 consecutive VLBW infants in a prospective, observational, clinical study. Inclusion criteria were GA $<32 \mathrm{wk}$ and birth weight of $605-1,500 \mathrm{~g}$. These infants were admitted to the Neonatal Intensive Care Unit of the Children's Hospital, Helsinki University Hospital, between June 2007 and November 2008. All studies were performed with the approval of the institutional review board of Helsinki University Hospital. An informed consent was obtained from the parents.

Patient characteristics are shown in Table 3. All mothers received one or two doses, $12 \mathrm{mg}$ each, of ante partum betamethasone treatment at 12 - to 24 -h intervals. Criteria for preeclampsia $(n=16)$ were elevated blood pressure and proteinuria (23). At delivery, the attending obstetrician made the diagnosis of clinical chorioamnionitis $(n=15)$.

A control population for cord plasma samples and cord HLA-DR samples on days 1 and 3 comprised 25 healthy infants with GA $>34 \mathrm{wk}$.

\section{Postnatal Morbidity}

As an index of neonatal morbidity, we used the SNAP-II score. SNAP-II includes mean arterial pressure, urine output, $\mathrm{PaO}_{2} / \mathrm{FiO}_{2}$, seizures, blood $\mathrm{pH}$, and temperature calculated for the first $12 \mathrm{~h}$. The extended version, SNAP Perinatal Extension-II, is an index for neonatal mortality, taking into account perinatal factors: birth weight, being small for $\mathrm{GA}$, and Apgar score at $5 \mathrm{~min}$ (24). We also used $\mathrm{PaO}_{2} / \mathrm{FiO}_{2}$ for the first $12 \mathrm{~h}$ as an independent marker for early respiratory morbidity.

The attending clinicians, blind to the study, made the diagnosis of RDS, defined as the infants showing radiological evidence of RDS as described by Edwards et al. (25) and requiring respiratory support at $24 \mathrm{~h}$ after birth. Of the 56 infants, 35 were classified as RDS infants and 21 as NoRDS infants. Three of the NoRDS infants required no respiratory support at $24 \mathrm{~h}$, and the remaining 18 were on nasal continuous positive airway pressure.

Of VLBW infants, 41 (73\%) were intubated at birth for respiratory insufficiency and received surfactant (Curosurf, $100 \mathrm{mg} / \mathrm{kg}$; Cornerstone Therapeutics, Cary, NC) within $15 \mathrm{~min}$ after birth. BPD, defined as the need for supplemental oxygen at the age of 36 gestational weeks (26), was diagnosed in 16 infants. Of note, 15 of these infants belonged to the RDS group and 1 to the NoRDS group. Presence or absence of BPD could not be diagnosed in four infants because of death before the age of $36 \mathrm{wk}$.

IVH (27) was diagnosed with routine ultrasound scans on day 1,3 , or 7 in 19 (34\%) of the VLBW infants. Bilateral grade III/IV IVH was diagnosed in seven infants, and one infant had unilateral grade II/III IVH. The remaining 11 had either unilateral or bilateral grade I-II IVH.

All received benzylpenicillin 100,000 IU/kg twice daily and netilmycin $2.5 \mathrm{mg} / \mathrm{kg}$ twice daily from the first day of life for $2-5 \mathrm{~d}$. In eight infants, benzylpenicillin was changed to vancomycin during the first postnatal week. The indications for vancomycin (in one infant each) were clinical suspicion of a septic infection, high C-reactive protein, perforation of the bowel, and pathologic distension of the bowel (no infection). Two infants each had a positive blood culture for Staphylococcus epidermidis and surgical closure of patent ductus arteriosus (no infection).

No infection developed in 25 infants (45\%). Infants were classified as having infection, when the attending clinicians, blind to the study, made the diagnosis of infection on the basis of symptoms and signs, leukocytes, and C-reactive protein. In all these patients, blood cultures were performed and antibiotics instituted. Of the 31 blood cultures, 17 were positive. In two infants, blood culture was positive on the day of birth (Escherichia coli and Bifidobacteria). Infections appeared late (after $72 \mathrm{~h}$ ) in all but those two infants, and in all but six infants, infection appeared after $7 \mathrm{~d}$ (Figure 4).

Patent ductus arteriosus was treated by administering indometha$\operatorname{cin}(n=23)$ or ibuprofen $(n=4)$. In two infants, patent ductus arteriosus was closed surgically as a primary treatment, and in seven infants, surgery was used after failure of pharmacological treatment.

Six infants died: one each because of lung hypoplasia at day 1 , severe IVH at $4 \mathrm{~d}$, necrotizing enterocolitis at $11 \mathrm{~d}$; two died of persistent infection (at $18 \mathrm{~d}$ and at $6 \mathrm{wk}$ ); and one died of severe BPD at 3 mo of age.

\section{Blood Samples}

Cord blood was drawn at birth from the umbilical vein with a pyrogen-free syringe. Postnatal samples were taken through an arterial cannula concurrently with clinical samples at day 1 (within $24 \mathrm{~h}$ ), day $3(72 \pm 12 \mathrm{~h})$, and day $7(5-7 \mathrm{~d})$. In infants with birth weight of $751-$ $1,000 \mathrm{~g}$, research blood samples $(1.8 \mathrm{ml})$ were drawn simultaneously with a red-cell transfusion given on clinical indication. If birth weight was $500-750 \mathrm{~g}$, samples were drawn only for flow cytometry $(0.4 \mathrm{ml})$. In control infants, blood samples for flow cytometry were obtained concurrently with samples from the heel stick required clinically, and cytokines were determined only in cord blood samples.

After being drawn, blood samples were immediately divided into two or three aliquots. For study of monocyte surface markers with flow cytometry, a $0.4-\mathrm{ml}$ aliquot was added to a pyrogen-free tube containing citrate phosphate dextrose (acid citrate dextrose; Baxter Health Care, Norfolk, UK) and was immediately cooled to $0^{\circ} \mathrm{C}$ and kept until analysis in an ice-water bath to minimize cell activation. For determination of cell surface HLA-DR on monocytes, samples were stained within $6 \mathrm{~h}$, kept on ice, and analyzed within $24 \mathrm{~h}$ (7).

Blood for plasma samples was added to $3.8 \%$ sodium citrate tubes (Becton Dickinson (BD), Franklin Lakes, NJ). Plasma was separated by centrifugation at $2,500 \mathrm{~g}$ for $15 \mathrm{~min}$ at $+4^{\circ} \mathrm{C}$ without delay, immediately frozen, and stored at $-80^{\circ} \mathrm{C}$.

\section{Determination of HLA-DR Expression by Flow Cytometry}

The method for determination of monocyte HLA-DR expression was modified from the method of Repo et al. (7). Monocytes in 25- $\mu$ l aliquots of whole blood were double labeled with CD14 fluorescein isothiocyanate antibodies (BD) and HLA-DR R-phycoerythrin-conjugated antibodies (BD). Mouse immunoglobulin- $\gamma 1$ phycoerythrin served as a control (BD). After labeling, contaminating erythrocytes were lysed by addition of $2 \mathrm{ml}$ ice-cold fluorescence-activated cell sorting lysing solution (BD). After a 3-min incubation on ice, the leukocytes were centrifuged for $5 \mathrm{~min}$ at $4^{\circ} \mathrm{C}$ at $400 \mathrm{~g}$. A second incubation with $2 \mathrm{ml}$ of fluorescence-activated cell sorting lysing solution was carried out for $5 \mathrm{~min}$ at room temperature. After centrifugation, the cells were resuspended in $1 \%$ formalin at $0^{\circ} \mathrm{C}$. A BD FACSort flow cytometer and CellQuest analysis software (BD) were used for acquisition and analysis of data. Monocytes were identified by CD14 positivity and on the basis of their light-scattering properties. In each sample, 2,000 monocytes were recorded. HLA-DR expression (HLA-DR\%) is reported as the percentage of HLA-DR-positive monocytes (the proportion of monocytes staining $>95 \%$ of that observed with the negative control) $(7,8)$. For the sake of fluency, the term "HLA-DR expression" is used to indicate the measured proportion of HLA-DR-positive monocytes relative to the whole monocyte population throughout the study. Therefore, the variability in absolute monocyte counts and the variability of the amount of HLA-DR on individual cells are not taken into account. Moreover, the fluorescence-activated cell sorting analyses were carried out on a single concentration of the labeling antibody, thus overlooking the potential effect of analytical conditions on the sensitivity of the assay.

CD11b was labeled with phycoerythrin-conjugated antibodies (BD) in a similar manner as HLA-DR. CD11b is reported as the median of molecules per cell (QuantiCalc; BD).

\section{Determination of Cytokines in Plasma}

Cytokines were analyzed with the Luminex technique. The test kit MILLIPLEX MAP High Sensitivity Human Cytokine Panel Premixed 13 Plex (Millipore, Billerica, MA) measured 13 different 
cytokines (IL-1 $\beta$, IL-2, IL-4, IL-5, IL-6, IL-7, IL-8, IL-10, IL-12, IL-13, interferon- $\gamma$, granulocyte macrophage colony-stimulating factor- $\beta$, and tumor necrosis factor- $\alpha$ ).

\section{Data Analysis}

Data were analyzed with SPSS 19.0 software (SPSS Inc., Chicago, IL). Patient data are presented as mean \pm SD and results as medians and ranges. Data not normally distributed required Friedman, MannWhitney, Kruskal-Wallis, and Spearman correlation tests. Linear regression models allowed statistical evaluation of correlations, and we did normalizing transformation (HLA-DR data reflect square root). Receiver operating characteristic analysis was performed for HLA-DR on day 3.

$P$ values of $<0.05$ were considered statistically significant. Power analysis was performed for patients with and without RDS. With sample sizes of 20 NoRDS infants and 35 RDS infants, a difference of 0.8 SD was detectable at a power of 0.8 .

The ethical requirements for different sample volumes for various birth weight categories, and the additional requirement of concurrent blood transfusion in the smallest infants, resulted in protocol-associated, but individually varying, missing values.

\section{SUPPLEMENTARY MATERIAL}

Supplementary material is linked to the online version of the paper at http:// www.nature.com/pr

\section{STATEMENT OF FINANCIAL SUPPORT}

This work was supported by the Sigrid Jusélius Foundation, Finska Läkaresällskapet, the Foundation for Pediatric Research (Helsinki, Finland), and a special governmental subsidy for health sciences research.

Disclosure: There are no financial relationships relevant to this article to disclose.

\section{REFERENCES}

1. Clapp DW. Developmental regulation of the immune system. Semin Perinatol 2006;30:69-72.

2. Hallwirth U, Pomberger G, Pollak A, Roth E, Spittler A. Monocyte switch in neonates: high phagocytic capacity and low HLA-DR expression in VLBWI are inverted during gestational aging. Pediatr Allergy Immunol 2004;15:513-6.

3. Yerkovich ST, Wikström ME, Suriyaarachchi D, Prescott SL, Upham JW, Holt PG. Postnatal development of monocyte cytokine responses to bacterial lipopolysaccharide. Pediatr Res 2007;62:547-52.

4. Frazier WJ, Hall MW. Immunoparalysis and adverse outcomes from critical illness. Pediatr Clin North Am 2008;55:647-68, xi.

5. Adib-Conquy $\mathrm{M}$, Cavaillon JM. Compensatory anti-inflammatory response syndrome. Thromb Haemost 2009;101:36-47.

6. Volk HD, Reinke P, Krausch D, et al. Monocyte deactivation-rationale for a new therapeutic strategy in sepsis. Intensive Care Med 1996;22:Suppl 4:S474-81.

7. Mentula P, Kylänpää-Bäck ML, Kemppainen E, et al. Decreased HLA (human leucocyte antigen)-DR expression on peripheral blood monocytes predicts the development of organ failure in patients with acute pancreatitis. Clin Sci 2003;105:409-17.

8. Mentula P, Kylänpää ML, Kemppainen E, et al. Plasma anti-inflammatory cytokines and monocyte human leucocyte antigen-DR expression in patients with acute pancreatitis. Scand J Gastroenterol 2004;39:178-87.

9. Turina M, Dickinson A, Gardner S, Polk HC Jr. Monocyte HLA-DR and interferon-gamma treatment in severely injured patients-a critical reappraisal more than a decade later. J Am Coll Surg 2006;203:73-81.
10. Livingston DH, Appel SH, Wellhausen SR, Sonnenfeld G, Polk HC Jr. Depressed interferon gamma production and monocyte HLA-DR expression after severe injury. Arch Surg 1988;123:1309-12.

11. Monneret G, Lepape A, Voirin N, et al. Persisting low monocyte human leukocyte antigen-DR expression predicts mortality in septic shock. Intensive Care Med 2006;32:1175-83.

12. Allen ML, Peters MJ, Goldman A, et al. Early postoperative monocyte deactivation predicts systemic inflammation and prolonged stay in pediatric cardiac intensive care. Crit Care Med 2002;30:1140-5.

13. Jaarsma AS, Braaksma MA, Geven WB, van Oeveren W, Bambang Oetomo $\mathrm{S}$. Activation of the inflammatory reaction within minutes after birth in ventilated preterm lambs with neonatal respiratory distress syndrome. Biol Neonate 2004;86:1-5.

14. Nupponen I, Pesonen E, Andersson S, et al. Neutrophil activation in preterm infants who have respiratory distress syndrome. Pediatrics 2002;110(1 Pt 1):36-41.

15. Birle A, Nebe CT, Gessler P. Age-related low expression of HLA-DR molecules on monocytes of term and preterm newborns with and without signs of infection. J Perinatol 2003;23:294-9.

16. Kanakoudi-Tsakalidou F, Debonera F, Drossou-Agakidou V, et al. Flow cytometric measurement of HLA-DR expression on circulating monocytes in healthy and sick neonates using monocyte negative selection. Clin Exp Immunol 2001;123:402-7.

17. Turunen R, Nupponen I, Siitonen S, Repo H, Andersson S. Onset of mechanical ventilation is associated with rapid activation of circulating phagocytes in preterm infants. Pediatrics 2006;117:448-54.

18. Monneret G, Finck ME, Venet F, et al. The anti-inflammatory response dominates after septic shock: association of low monocyte HLA-DR expression and high interleukin-10 concentration. Immunol Lett 2004;95: $193-8$.

19. Abe R, Hirasawa H, Oda $\mathrm{S}$, et al. Up-regulation of interleukin-10 mRNA expression in peripheral leukocytes predicts poor outcome and diminished human leukocyte antigen-DR expression on monocytes in septic patients. J Surg Res 2008;147:1-8.

20. Pachot A, Lepape A, Vey S, Bienvenu J, Mougin B, Monneret G. Systemic transcriptional analysis in survivor and non-survivor septic shock patients: a preliminary study. Immunol Lett 2006;106:63-71.

21. Fumeaux T, Pugin J. Role of interleukin-10 in the intracellular sequestration of human leukocyte antigen-DR in monocytes during septic shock. Am J Respir Crit Care Med 2002;166:1475-82.

22. Koppelman B, Neefjes JJ, de Vries JE, de Waal Malefyt R. Interleukin-10 down-regulates MHC class II alphabeta peptide complexes at the plasma membrane of monocytes by affecting arrival and recycling. Immunity 1997;7:861-71.

23. ACOG Committee on Obstetric Practice. Practice bulletin \#33: diagnosis and management of preeclampsia and eclampsia. Obstet Gynecol 2002;99:159-67.

24. Richardson DK, Corcoran JD, Escobar GJ, Lee SK. SNAP-II and SNAPPE-II: Simplified newborn illness severity and mortality risk scores. J Pediatr 2001;138:92-100.

25. Edwards DK, Hilton SV, Merritt TA, Hallman M, Mannino F, Boynton BR. Respiratory distress syndrome treated with human surfactant: radiographic findings. Radiology 1985;157:329-34.

26. Bancalari E, Claure N. Definitions and diagnostic criteria for bronchopulmonary dysplasia. Semin Perinatol 2006;30:164-70.

27. Papile LA, Burstein J, Burstein R, Koffler H. Incidence and evolution of subependymal and intraventricular hemorrhage: a study of infants with birth weights less than 1,500 gm. J Pediatr 1978;92:529-34. 